\title{
Effect of Soliman Auricular Allergy Treatment (SAAT) on IgE-mediated Reactions Due to Exposure to Mammalian Meat Oligosaccharide, Galactose- $\alpha-1,3-$ Galactose
}

\author{
Donald Liebell \\ The Liebell Clinic, Virginia Beach, Virginia, USA
}

Email address:

necksecret@gmail.com

\section{To cite this article:}

Donald Liebell. Effect of Soliman Auricular Allergy Treatment (SAAT) on IgE-mediated Reactions Due to Exposure to Mammalian Meat Oligosaccharide, Galactose- $\alpha-1,3-$ Galactose. American Journal of Biomedical and Life Sciences.

Vol. 8, No. 5, 2020, pp. 189-197. doi: 10.11648/j.ajbls.20200805.18

Received: September 1, 2020; Accepted: September 17, 2020; Published: September 23, 2020

\begin{abstract}
Allergy to galactose 1,3, $\alpha$-galactose (alpha gal) - a natural cellular carbohydrate component of non-catarrhine primate mammalian muscle tissue - is a recognized and prevalent disorder. No effective treatment has been established beyond palliative and emergency treatment medications used for food allergies, in general. This tick-triggered illness can affect humans, not only through mammalian meat consumption, but via exposure to numerous food and non-food products containing alpha gal, as well as skin contact and inhalation of airborne particulates. The objective was to document with a statistically powerful cohort, the efficacy of the ear acupuncture procedure, Soliman Auricular Allergy Treatment (SAAT), in support of the neurological theory of allergy. SAAT procedures involved electrical detection of case-specific auricular acupuncture points within the ovoid region designated as Soliman Allergy Zone (SAZ). 3mm-long, semi-permanent, intradermal ear acupuncture needles were inserted as indicated - secured by surgical tape and medical adhesive to enable ongoing stimulation for 3 weeks. Participants removed the acupuncture needles at home. Patients reported diverse histories of typical allergic symptoms, ranging from mild gastrointestinal or pruritic symptoms to severe anaphylactic reactions, as well as atypical symptoms such as joint problems, fatigue, anxiety, and sleep dysfunction. Primary outcome was patients' written attestation of restored ability to be exposed to alpha gal without allergic incident, or significant reduction. Among the initial 155 patients providing follow up data, 147 patients reported some degree of returned consumption of mammalian meat without allergic incident, or significant reduction in severity or frequency. The paradigm-shifting relevance of this preliminary investigation-successfully treating a globally-relevant and problematic arthropod vector-triggered allergy, cannot be overestimated. No prophylactic or supportive treatment for AGA has been previously reported. The response was of groundbreaking statistical significance; the success rate for this first wave of follow-up responding participants who completed the treatment was $94.8 \%$.
\end{abstract}

Keywords: Alpha Gal, SAAT, Auricular Medicine, Ear Acupuncture, Tick-Borne Illness

\section{Introduction}

Awareness of allergy to the non-catarrhine primate muscle oligosaccharide, galactose- $\alpha$-1,3-galactose (alpha gal) has been escalating. Akin to other food allergens, exposure to alpha gal can provoke dermatological, gastrointestinal, neurological, and respiratory consequences, including anaphylaxis. This clinical investigation, however, provides additional insights into other symptoms not likely considered. Those afflicted with alpha gal allergy (AGA) may be oblivious to a specific encounter with a hematophagous ectoparasite which provoked anti- $\alpha$-gal IgE-antibody production, let alone its sequelae. The bite of the lone star tick (Amblyomma americanum) - the arachnid primarily associated with triggering AGA, is not presumed to elicit the diagnostically indicative erythema migrans reaction sporadically observed in association with Lyme disease-spreading Ixodes scapularis ticks. However, a history of delayed-onset allergic reactions several hours after eating mammalian meat (or other routes of alpha gal exposure), is considered pathognomonic.

The association between tick bite and mammalian meat allergy was initiated in the 1980 s by Sheryl van Nunen, in 
Australia, albeit unpublished until decades later [1]. Most research has focused on risks of developing AGA and monitoring IgE levels. Mammalian meat allergy was first recognized in the United States in 2006, as an unanticipated consequence of Thomas Platts-Mills' clinical trials for the cancer drug, cetuximab (marketed in 2004). Cases of anaphylaxis were determined to be caused by a mouse-derived protein and alpha gal molecules in the drug. Coincidentally, Platts-Mills' insights into the tick component came about when he developed the allergy after hiking [2]. In 2009, Commins et al reported the atypical (to food allergy) delayed onset of symptoms 3-6 hours after consumption of red meat, presently known to be notorious for alpha gal allergy [3].

It is abundantly clear that ticks cause human illness by multiple mechanisms. The correlation between rising tick populations and AGA prevalence has been considered. However, it might be more likely a function of heightened awareness of a long-existing, but unconsidered entity. Chabris and Simon coined the term inattentional blindness for failures of awareness resulting from focusing attention to what we believe is important, while filtering what needs to be seen. Focusing attention on one thing blocks human ability to see the otherwise obvious [4]. The perception of a rising incidence of AGA is plausibly in reverberation to amplified public awareness, medical insights to its epidemiology, combined with increased prevalence and persistence, concomitant with the absence of prophylactic or corrective treatment. Alpha gal allergy is easy to detect, now that we know to look for it. More importantly, with Soliman Auricular Allergy Treatment (SAAT), it appears prophylactic treatment is available.

\section{Current Approaches}

Allergen-specific immunotherapy (AIT) has been extoled as the only causal treatment of allergic diseases. Its effectiveness aside, its mechanisms (as well as those of allergic diseases) remain unclear; this is the subject of detailed studies worldwide [5]. Ring and Gutermuth profess a substantial need for research to elucidate the currently unknown mechanisms of AIT [6]. Prior to this publication, little evidence of a reliable, efficacious, prophylactic, or corrective treatment has been presented. Burks and Wesley et al hold that to date, there are no regulatory approved treatments for food allergy [7]. Potentially life-threatening food allergies affect nearly 6 million U.S. children, but no treatment has been approved by the Food and Drug Administration (FDA).

\subsection{Acute and Emergency Symptomatic Treatment}

According to the American Academy of Allergy, Asthma \& Immunology (AAAAI) alpha gal allergy is treated for acute symptoms in the same urgent care manner as any other food allergy — with anti-histamines, epinephrine, and other medications, dictated by the presence and severity of symptoms [8]. Pharmaceutical intervention only manages symptoms of acute allergic reaction; the underlying immune disorder goes unaddressed. A paucity of self-limiting cases of
AGA has been cited, with the possibility of desensitization over a period of years, with restored tolerance achieved via gradual reintroduction in tiny doses [9]. Some have suggested the potential for restoring tolerance to alpha gal by avoiding ticks for 1-2 years or through small dose exposure to the allergen [10].

\subsection{Global Urgency for a Prophylactic Intervention}

$\mathrm{Yu}$, et al suggest the rising prevalence of food allergies has not resulted in an improved standard of care [11]. It is clear that after more than a decade of pursuit of insights to AGA, the consensus among researchers is that the only prophylaxis remains to be strict avoidance of mammalian meat, its by-products, and other $\alpha$-Gal-containing foods. Practical feasibility to avoid this offending oligosaccharide within the context of daily human life is questionable. Its dubious classification as merely a food allergy trivializes its complexity and inadequately defines its perils. Alpha gal is an omnipresent component of many foodstuffs and non-food products, thus posing considerable hazard to those allergic to it. The massive potential for unintentional exposure, even from infinitesimal quantities cannot be overestimated. For some, momentary inhalation of fumes of mammalian meat cooking, or contact with infinitesimal quantities of the carbohydrate can provoke an allergic response. Therefore, victims of AGA would benefit from any treatment capable of producing any degree of desensitization. The allergy has been reported by patients to drastically reduce quality of life not only for themselves, but their families, and social groups. Such parties have reported considerable desire and motivation for achieving resolution of this tick-triggered illness. Immunity towards inadvertent and unknowing exposures would radically improve quality of life.

\subsection{Diagnosis of Alpha Gal Allergy}

Allergy as a medical discipline, is relatively fledgling; its acceptance historically, was far from immediate. Serious scientific inquiry, specifically into food allergies has been relatively recent, although Hippocrates (460-377 BC), referred to "hostile humors," known to modern medicine as IgE antibodies which caused symptoms following consumption of cheese [12]. The term, allergy was coined in 1906 by the Austrian pediatrician Clemens von Pirquet. According to Sampson, in the 1980s, there was little public awareness of allergy. Skepticism of it as a diagnosis existed; some researchers did not consider the field to be an authentic science. The early 1950 s discovery of mast cells was a major boost to its understanding, but it was not until the late 1960s that IgE was discovered. Its clinical application by allergists began in the mid-1990s [13].

History and examination are widely considered the primary tools for food allergy diagnosis. Unlike the rapid onset of symptoms characteristic of typical food allergy reactions, those of alpha gal do not generally present for many hours after exposure. Telltale patterns are not always consistent, nor is the patient always cognizant of the causative agent as the 
culprit. Some clinic patients reported rapid onset of symptoms or biphasic reactions more commonly associated with typical food protein-based allergies.

IgE serology has become an essential component for AGA diagnosis. A strong case can be made for its inclusion as part of general and routine medical evaluation, as it is a very inexpensive route towards potentially invaluable feedback. However, IgE levels do not appear to be directly proportional to severity of alpha gal-related allergic reaction or degree of sensitivity. One may have significantly high IgE response yet exhibit no allergy symptoms. Conversely, some of the most severe cases can be those exhibiting very low levels. In many cases, patients report a definitive pattern of cause and effect, prompting the necessity for confirming serology. In other cases, the alpha gal-savvy practitioner requisites IgE testing in suspicion of its presence; the patient has not considered a connection between meat consumption and delayed allergic reaction. Provocative tests complement IgE serology. Skin prick tests are potentially useful, although they may not correlate with symptoms. According to Lopez and Mendez, around $60 \%$ of positive tests do not reflect symptomatic allergy to a specific food [14]. Gellersted, Bengtsson, and Niggemann contend oral provocation is the most accurate means of diagnosis for clinically relevant IgE-associated food allergies. Elimination diets and re-introduction of foods, or open oral challenges are suggested. A double-blind, placebo-controlled food challenge is the gold standard for antigen identification [15]. Loh and Tang cite a lack of high-quality evidence based on oral food challenges to determine food allergy prevalence, designating $\operatorname{IgE}$ and clinical history as surrogate measures for diagnosis [16]. Although oral food challenge is reliably diagnostic, its feasibility and safety outside of specially-equipped and staffed facilities for management of anaphylaxis limits its implementation [17]. It is further problematic in the diagnosis of AGA due to the commonly delayed nature of the reaction.

\section{A Revolutionary Approach to Food Allergy}

According to Igea, Pirquet's original meaning of allergy was corrupted, limiting it to primarily IgE-mediated illnesses [18]. In 2018, Kanagaratham et al remarked that expanding the research toolbox of experimental food allergy is important for our understanding of its mechanisms, including availability of additional experimental models to discover new intervention points for its treatment [19]. SAAT could not be more precisely befitting. The exclusively biochemical model of medicine has prevailed since the nineteenth century (despite Chinese acupuncture predating it by millennia). The neurological (bioenergetic) model of allergy is based upon the perspective that human beings are complex networks of energy fields that coexist with the physical cellular systems. The electromagnetic forces associated with biochemical processes must not be ignored. Integration of physics and chemistry into medicine creates a complete system. In 1937,
Harold Burr, PhD (1889-1973), Professor Emeritus of Anatomy, Yale University Medical School determined that abnormal voltage gradients preceded abnormal cellular growth such as cancer. He foresaw the link between physics, chemistry, and biology, which would eventually be validated via applications of ECG, EMG, and other electrophysiological technologies. Burr determined that electromagnetic properties are the organizing force of homeostasis. He insisted electrodynamic fields which control the human organism are signposts to the most promising trail that future explorers can follow. A prime example is cardiac diagnosis. Biochemistry and electrophysiology are both important and complementary diagnostic indicators, combined with auscultation. Serology alone may be insufficient. Neuro-electrical feedback can be critical; a solely chemical approach can be disastrous. Electromagnetic forces can influence chemical processes. Burr predicted that extensive application of attention to living electric fields in medicine would not be quickly embraced, despite no technical reason for such. This was exemplified by the sluggish, decades-long embracement of the electrocardiogram into mainstream medicine [20].

\subsection{Ear Acupuncture}

In 1956, Paul Nogier, MD of France (1908-1996) introduced a new medical discipline focused on the bioenergetic properties of the body: the ear acupuncture microsystem. Measurable electrical conductivity patterns observed on the auricle correlate with the embryological cellular patterns of tissue and organ development. Nogier's original inverted fetus ear homunculus is a cartograph of neurological activity that reflects dysfunction of organs and tissues. These projections do not manifest as fixed or anatomically generic acupuncture points (as per traditional Chinese medicine's meridian-based systems); they exist within zones possessing constantly changing and dynamic borders within the individual's unique auricular topography. These zones also overlap. Energetic disturbance of a bodily structure results in the production of an electrically active point, detectable by instrument within its physiologically correlating auricular projection zone.

Most importantly, Nogier discovered that stimulation of detected acupuncture points elicited health benefits. Armed with the understanding that the brain and body have neurological/embryological energy connections that can be observed, studied, and mapped; he developed a remarkable means to reduce human suffering and improve health through stimulation of the ear, based on somatosensory principles. His ear acupuncture (auricular therapy or auriculotherapy) became an internationally recognized approach, acknowledged by Hiroshi Nakajima (1928-2013), Director-General, World Health Organization. In his 1990 address, Dr. Nakijama acknowledged the debt modern medicine owes to Dr. Paul Nogier for his development of auricular therapy, recommending promotion of its usage, teaching, and further research worldwide [21].

In 2014, Nader Soliman, MD published case studies 
documenting the effects of Soliman's Auricular Allergy Treatment (SAAT)-his now patented ear acupuncture procedure (S224-0002US). He reported significant results in prophylaxis of numerous allergies within 3 weeks of a single treatment. In 2013, Soliman presented his success with over 600 cases of allergies at the Symposium of the American Academy of Medical Acupuncture that year. Paul Nogier's original auricular homunculus cartography designated a zone in the ear physiologically correlated with the liver. Through years of clinical observation, Soliman discovered that precise stimulation of acupuncture points (electrically detected within an expanded border of Nogier's liver zone), consistently triggered natural auto-resolution of allergic reactions [22].

\subsection{Mechanism of SAAT}

The auricle is innervated by cranial nerves $5,7,9$, and 10 , as well as the second and third cervical roots and cervical sympathetic plexus. Evidently, SAAT works via an embryological/neurological mechanism, which triggers restored auto-regulation of the neuroelectric dynamics of the liver [22]. Numerous physiological phenomena cannot be fully explained by their anatomical relationships. For example, referred pain patterns are evidence of physiological manifestations of bioenergetic embryological derivations. It has been established that acupuncture points are evidence of electrophysiological signals driven by internal organs; they can reflect the health condition of internal organs effectively, thus providing additional means to diagnosing diseases [23].

Recent studies have demonstrated health benefits derived from electrical stimulation to general regions of the auricle. Transcutaneous Auricular Vagal Nerve Stimulation (TAVNS) has been of medical interest. Mercante et al has lauded it as neuromodulation or neurostimulation techniques which are scientifically-validated, non-invasive, bottom-up brain modulation techniques, which could transpire to be the most affordable target for non-invasive manipulation of central nervous system functions [24]. Badran et al refer to vagal nerve stimulation as an emerging neuromodulatory technique capable of affecting efferent projections of the vagus nerve to target every major organ on the body with wide effects on bodily function [25]. These studies' conclusions certainly reaffirm Nogier's work from decades prior. Indeed, they provide insights to the mechanisms and medical potential for SAAT, although they are devoid of appropriate reference to ear acupuncture research and citations for the pioneering work of Nogier, Soliman, and others. Nevertheless, the likelihood of a vagal cranial nerve pathway, to at least partially explain the mechanism by which SAAT works, seems strong; Soliman Allergy Zone is innervated by the vagus nerve. However, general auricular vagus nerve stimulation is not the mechanism of SAAT. Successful treatment is predicated upon precise, skillful, and meticulous evaluation procedures and needle placement, which varies substantially from patient-to-patient. This is not based upon fixed acupuncture points, as per meridian-based traditional body acupuncture. Curiously, Soliman's clinical correlations are also congruent with the phenomenon of liver transplant-acquired allergies and the passive transfer of $\operatorname{IgE}$, not observed via transplant of other organs [26].

Neurological patterns associated with homunculi have been established over many decades of study with various experiments. Penfield and Boldery's 1937 study described somatotopic and somatosensory organization of the human brain [27]. Modern diagnostic technology continues to confirm their findings. Electrical stimulation of a specific area of the brain can produce movement or tingling sensations in the corresponding part of the body [28]. Kroeuning and Oleson's 1980 double-blind study at UCLA School of Medicine verified statistically significant accuracy of utilizing auricular diagnosis for musculoskeletal problems. Its results supported the hypothesis of the existence of a somatotopic organization of the body projected to the auricle [29]. A 2001 study further confirmed the established principle of mapping of the ear with magnetoencephalography (MEG) and functional MRI (fMRI). Comparisons of electrical activity to evaluate changes in blood flow confirmed that stimulating parts of the ear affected areas of the neck and face [30]. fMRI studies have provided evidence for proving the effects of auricular therapy on the brain [31]. A greater understanding of the depths of auricular therapy is ripe for ongoing medical study. Its benefits have been documented through decades of medical research, although in this author's opinion, gravely insufficiently publicized; it is worthy of greater research effort and funding, considering its superb clinical merits, safety, cost-effectiveness, and vast potential. Prior to Soliman, a paucity of research had been dedicated towards the neuroelectric theory of allergy. Although traditional Chinese medicine (TCM)-based acupuncture has been administered - attempting to alleviate allergies; no consistent, reproducible, and large-scale benefits have been documented. Paul Nogier's designated auricular acupuncture allergy zone (or point) was not sufficiently effective [22]. Years prior to this study, Soliman and Liebell observed independently, in their respective practices, resolution of mammalian meat allergy via application of SAAT, without specific awareness of alpha gal's tick-borne biochemical mechanism. Thus, success was anticipated in its precise application to AGA.

\section{Methodology}

The Centers for Disease Control indicate widespread prevalence of AGA in the southeastern United States, making the clinic's Virginia Beach location suitable for treating and documenting cases for preliminary investigation. Due to the public's urgency and demand for effective treatment; patients eagerly sought treatment, beginning in June of 2019, ongoing. Patients were not chosen or solicited; they privately and independently requested the services, largely in response to social media and word-of-mouth referral. Thus, randomization was inherent. HIPPA and informed consent forms were designed and provided by the Liebell Clinic, as per routine procedure. 


\subsection{Inclusion and Exclusion Criteria}

Patients aged 4-85, presented with an unambiguous history of symptoms attributable to alpha gal allergy. Most were previously diagnosed by an allergist with $\mathrm{IgE}$ levels greater than $0.35 \mathrm{kUA} / \mathrm{L}$. Notably, however, SAAT is not dependent upon IgE levels, food challenge, or skin prick tests. One criterion for inclusion was patient awareness of objective reactions to consumption and/or exposure to alpha gal, such as hives, gastrointestinal sequelae, respiratory distress, visible swelling, and anaphylaxis. History of subjective symptoms such as headache, fatigue, and other symptoms consequential to alpha gal exposure were considered. Most cited no awareness of difficulties with mammalian meat or alpha gal-containing products prior to onset.

Exclusion criteria for implementing SAAT were individuals with genetic and chromosomal disorders, history of nerve healing challenges, life-threatening conditions, organ transplant, cases of anticoagulation therapy and bleeding tendencies. Electrical stimulation was contraindicated for patients with cardiac pacemakers. Allergic reactions were not provoked intentionally or otherwise; exposure to alpha gal was not necessary or implemented. Patients were explicitly directed to maintain existing emergency medical supports such as antihistamine medications and epinephrine injectors, as per prescribing physician's direction; at no time were participants suggested to discontinue or reduce their usage.

\subsection{Examination}

Examination for Soliman Auricular Allergy Treatment was conducted using tools and methodologies developed by Nader Soliman, MD. SAAT is a medical innovation based upon Paul Nogier, MD's basic auricular medicine bio-energetic techniques developed in the 1960s (not to be confused with auricular therapy). No generic ear acupuncture "allergy point" exists. A strict and meticulous investigative procedure was required to determine the location of the appropriate corresponding electrically active auricular acupuncture point, which was different in every individual patient. This was accomplished by following the patented procedural criteria of SAAT.

\subsection{Interventions}

Seirin Spinex sterilized stainless-steel ear acupuncture intradermal needles $(3 \mathrm{~mm})$ were inserted according to SAAT methodology, under aseptic conditions. The inserted needle's ring handle remains visible, securing it, and enabling easy removal. Mastisol medical adhesive was used in conjunction with bandage tapes to secure the needles in order to maintain continuous stimulation for 3 weeks.

\subsection{Outcome Measures}

It is inherent within the parameters of this preliminary investigation that objective responses are challenging to quantify. SAAT's primary measure of efficacy was patients' direct reporting to the clinic their restored capacity to undergo exposure to alpha gal (intentional and otherwise) without allergic incident, or significant reduction. For many participants, treatment success was evident by lack of symptoms previously experienced throughout daily life. Reporting of elimination of reactions to the fumes of mammalian meat cooking is an example. Quantifying a lack of allergic response is challenging outside a controlled clinical environment. The non-presence of visible skin reactions or diarrhea, for example can be observed. Many more symptoms cannot be observed or objectively quantified. For milder cases, on their own accord, many patients gradually reintroduced meat consumption-reporting no allergic consequences.

\subsection{Sample Size, Statistical Power and Methods}

Sample sizes require adequate statistical power to yield acceptable results. Worldwide, the incidence of AGA has not been established due to insufficient investigation. Furthermore, numerous patients reported prior encounters with physicians who showed callous disregard for the possibility of the condition, as well as patent antagonism to IgE testing. Rather than appropriately considering clinical presentation, many patients stated their intelligence or sanity was questioned. Therefore, it can be inferred that alpha gal allergy is vastly underreported. The probability distribution must be determined from the largest sample sizes possible. According to Jones, Carley, and Harrison, clinical studies entail studying a sample of patients exhibiting a specific characteristic rather than the whole population. Inferences for an entire population may be drawn from such a sample [32].

For this preliminary investigation, statistical power assessment is challenging because the effects of SAAT on alpha gal allergy have no other treatment for comparison; none have been medically offered beyond attempts to gradually reintroduce mammalian meat. The current medical standard for management of AGA is abstinence from exposure and avoidance of the tick vector - both ominously implausible measures. Additionally, clinical results are difficult to quantify (IgE and skin prick test findings do not correlate with clinical results), thus making power calculation problematic. This may be similarly arduous with double-blind placebo studies. Nevertheless, considering the lack of historical clinical data of improvement revealed by treatment other studies; one may consider the clinical responses of 147 of 155 patients reporting the clinical benefits derived from SAAT to be powerful and statistically notable. Analysis of results required attention to factors of patients' compliance, subjective reporting, and objective reporting of diminished or eliminated allergic reactions experienced after one or more months post-treatment. To consider treatment successful, patients were required to document restored capacity to be exposed to alpha gal without incident, or significant reduction. The sample size of 155 respondents revealing success of $94.8 \%$ was of revolutionary medical importance.

To date, more than 500 patients have received SAAT at the Liebell Clinic since commencing formal documentation for 
research purposes. However, it will take many months before all post-treatment feedback can be acquired. This report accounts for the feedback provided by the first 155 to provide written follow up documentation, regardless of outcome. Many of these patients reported prior to SAAT as being fume reactive to alpha gal. Adherence to treatment parameters was overall high. 141 of the participants retained their ear acupuncture needles for the required 3 weeks. 3 patients, who were unable to retain their needles, returned for re-application.

\section{Results: Assessment of Clinical Response}

All patients were instructed to continue avoiding exposure to alpha gal-containing foods and non-food products (as most had already been). Those who reported no harmful consequences of dairy were recommended to nevertheless avoid excessive consumption during the 3-week period and beyond. Participants with no history of anaphylaxis or other severe reaction, on their own accord elected to test eating some mammalian meat after the 3 -week SAAT period. They were instructed to eat only the smallest amount (about the size of a grain of rice) - without any sauces, spices, mixtures, preservatives, or other ingredients. It was recommended to wait a day (feeling no negative effects) before repeating the test, and to very gradually re-introduce mammalian meat and/or dairy, provided no allergic response was triggered.

Out of the 155 participants, 147 reported successful self-administered oral food challenge post SAAT. These participants reported resolution or diminished alpha gal-related symptoms and reactions within 2-6 months following initial SAAT auricular needle insertion. This also included elimination or significantly diminished reaction to fumes of mammalian meat cooking. The statistical significance is clear.

\subsection{Non-compliance and Results}

The 3-week period of SAAT within the Soliman Allergy Zone was the procedural requirement, determined through years of Nader Soliman's clinical observations. Thus, negative results reported for anything less than 3 weeks of retaining their SAAT needles would have been categorized as inconclusive, but not failed. However, despite a few cases of some degree of non-compliance; the results were extraordinary. This includes patients who reported intentional consumption of mammalian meat during the 3 weeks of retaining their SAAT needles, accidental consumption, as well admission of eating meat at fast food restaurants (vehemently discouraged at any time). Due to the stunning outcomes reported by this preliminary cohort, no need to delineate non-compliant participants individuals proved to be necessary. For future formal studies, greater scrutiny would be likely applied.

\subsection{Adverse Events}

SAAT acupuncture treatment was very well tolerated. A few patients reported minor tenderness at the site of needle insertion on the auricle, which subsided rapidly. One patient reported swelling, which subsided without complication; this individual reported resolution of AGA and has safely and comfortably consumed meat after a 5-year abstinence. One patient experienced post-treatment anxiety, which was easily resolved with needling a point detected in the auricular acupuncture projection zone of the amygdala (Nogier phase 1) [33]. One patient reported possible infection of the auricular cartilage (for which she received precautionary antibiotic treatment from her local physician). However, months later, she suggested it was not infection, but more likely an allergic response to the medical adhesive. It is uncertain what occurred. The clinic had never seen such an occurrence before through thousands of applications of semi-permanent ear acupuncture needles, adhesive, and tape, applied over many years.

\subsection{Limitations}

This was a private practice-conducted preliminary investigation. Documenting a statistically significant number of successful cases was intended to serve as a stepping-stone towards sanctioned clinical trials. Thus, several limitations were expected. Patients provided detailed history, and in many cases written reports revealing positive IgE serology. However, no provocative tests or other conventional diagnostic measures were taken. Some patients reported a history of anaphylaxis or other severe reactions to alpha gal. These patients were strictly warned against pursuing intentional consumption and exposure to the allergen following treatment. Such individuals were encouraged to monitor their lack of allergic reactions compared to what they previously reported as being frequently occurring due to inadvertent exposures. Pre- and post-treatment researcher-observed food challenging tests or IgE studies were not conducted. Such studies would likely enable reduction of variables of complicating additional allergens. Provocative evaluation and documentation would require the appropriate personnel and researchers licensed and experienced to administer tests, and to provide any necessary palliative or emergency medical treatment for intentionally induced allergic reactions. Perhaps such study will be considered based upon the paradigm-shifting significance of the success of this preliminary investigation. No data of race, ethnicity, blood type, or genetic factors were correlated. Formal studies could consider these and other variables, including complication of other concomitant food allergies. Critical to further investigation is monitoring potential reemergence of AGA from new tick bites. A few patients reported having been bitten by ticks sometime after successful SAAT, without apparent relapse. However, more data will be required over a long period of time for conclusive evidence.

Due to the 3-week time requirement for retaining SAAT needles, as well as abstinence from alpha gal exposure, perfect compliance has its challenges. Various reasons are possible for needle retention under 3 weeks. Moisture or oiliness of skin 
undermining tape bonding is possible. One might forget needles are present, and inadvertently pick at and dislodge them. Some patients may ignore the instructions of how one should reintroduce mammalian meat in the purest, smallest, and most gradual manner. Although highly discouraged, some patients may consume beef burgers from fast food restaurants, which are potentially laden with preservatives to which they may be reactive. Within the context of this preliminary investigation, it was in some cases, not possible to differentiate between adverse gastrointestinal, pruritic, or neurological effects reported by subjects suffering other existing allergies; the required controlled environment was not feasible. We did not have the means to monitor post-treatment allergic reactions that may have been attributable to too rapid or too large of a portion of meat re-introduction, as well as consumption of combinations of foods containing other allergens.

\section{Discussion}

In this phase of study, results from a statistically powerful cohort suggests SAAT rapidly, consistently, and effectively triggers natural AGA resolution. Participants' adherence level to the parameters of treatment was overall, high. $94.8 \%$ of respondents reported restored ability to withstand exposure to alpha gal without allergic reaction, or at least substantially diminished severity and/or duration. This primary analysis meets success criteria and statistical significance of outcomes to warrant further formal study. Follow-up evaluations will determine how long its effects are maintained. The possibility of AGA returning upon future tick bites must be considered and studied within the context of the SAAT evaluation and management paradigm. Soliman's 2014 study involving SAAT for numerous allergies suggests long-lasting resolution [22]. Prior to this private in-clinic investigation, patients of Soliman and Liebell (independently, at their respective facilities) have reported years of lasting relief from various other food allergies, including mammalian meat. It is acknowledged that this does not constitute proof for proclaiming specific longevity of its alpha gal allergy-resolving effect.

Within the context of this original preliminary investigative work, it would be desirable to discuss comparative research. However, none of such appears to exist. Leading medical authoritative institutions in the field of allergy and immunology have maintained the position that like other protein associated food allergies, alpha gal is managed solely by avoidance of the substance. To the best of the Liebell Clinic's knowledge, no treatment for alpha gal allergy has been subjected to a randomized, clinically controlled, double-blind placebo study. A research-based university hospital must be the setting for such study. Weeks prior to treatment, via oral food challenge, participants would have their allergic reactions intentionally provoked, witnessed, assessed, graded, and documented. Emergency medical facilities, staffing, and medications would be compulsory for palliative care for reactions, especially anaphylaxis.
Participant overnight stay would be required due to the broad range of reaction times. Weeks after participants' recovery from reactions, SAAT would be administered. A placebo group could receive ear acupuncture treatment at sham points.

The statistical measurement of superiority of an intervention versus placebo is a medical research convention has been used for evaluation of pharmacological and other products. However, patients of this investigation made it abundantly clear that intentional provocation of their allergic reactions would be inhumane and stunningly undesirable. Many expressed hypothetically, that tremendous financial compensation would be required to have their allergic reactions induced. While a double-blind placebo study is feasible to implement, perhaps the scientific establishment might reconsider its necessity as proof of AGA resolution via SAAT. Countless studies of various medical interventions have been conducted for treatment of maladies for which no objective visible or measurable evidence of their resolution is possible. It is arguable that hundreds of patients' subjective reporting (ongoing to date) of success with SAAT suffices as an ethical, humane, and cost-effective means to sufficiently prove its efficacy.

Exploratory outcomes for future SAAT investigation could be monitored through a compendium of diagnostic methods, including IgE, skin prick tests, oral food challenges, and patients' subjective reporting. No expectation or claim exists for SAAT's effect on reducing alpha gal IgE levels. SAAT's advanced auricular medicine techniques are not currently recognized as medically diagnostic. Likewise, $\operatorname{IgE}$ is a valuable indicator; it does not constitute proof of allergy or its resolution. Positive IgE can exist without clinical manifestation of allergy. Levels below or above 0.35 are not directly proportional to one's allergy severity and sensitivity. Although it is immensely valuable for initial diagnosis of AGA; fluctuations in IgE levels with or without alpha gal abstinence suggests its exclusion as a determinant of efficacy for SAAT. IgE levels would not likely be in direct proportion to the evident natural clinical resolution promoted by the treatment.

Established allergy authorities have represented emergency medical intervention, tick avoidance, and abstinence from mammalian meat consumption as the standard for prevention. Post SAAT feedback of clinical improvements such as reduction in headaches, fatigue, gastroesophageal reflux, joint pain, anxiety, as well as improved sleep (without any dietary or behavioral changes) are fascinating. This lends to the plausibility that particulates of alpha gal from airborne and surface sources could be ubiquitous to the point that alpha gal afflicted individuals suffer some degree of daily chronic symptoms from exposure to unavoidable sources. The plethora of symptoms (both typical and atypical to food allergies) reported to have been resolved or reduced by SAAT, support inclusion of alpha gal IgE screening in routine medical evaluation. This inexpensive test could potentially detect the cause of gastrointestinal symptoms reduce healthcare costs significantly, if performed prior to endoscopy and colonoscopy. These are tests cited by several studies to be performed 
excessively and with questionable clinical merit [34-36].

We may infer that countless millions of people worldwide suffer various chronic symptoms, oblivious to its arthropod vector etiology. Thus, the current medical standard of avoidance and abstinence serves as starkly insufficient precautionary and preventative advice. This reality fortifies the urgent demand for prophylactic support to facilitate the natural resolution of the condition. Sampson contends that despite the great advances in the field of food allergy, the remaining questions will likely keep investigators occupied for at least the next three decades [37]. The intention of work is to advance the neuro-electric model of allergy for the benefit of alpha gal allergy sufferers, worldwide.

Auricular acupuncture is hardly an experimental treatment, nor is its usage for symptomatic relief in question. Public awareness, interest, and enthusiasm for its application in support of AGA (since launching this study) has been very strong. Those afflicted with this tick-borne illness are clearly eager for the opportunity for its resolution. The clinical responses to SAAT makes the rationale for attention to this bioenergetic approach clear; it has been effective for a medical condition for which no treatment has been successful. The discovery and development of SAAT could transmogrify the evaluation and management of allergy.

\section{Conclusions}

Prior to this preliminary investigation, no prophylactic or corrective treatment has been demonstrated for this tick-triggered illness; thus, any level of success achieved would be groundbreaking. Thus, these results are of revolutionary significance. Among the first 155 patients providing follow-up data, $94.8 \%$ reported resolved or appreciatively diminished sensitivity to the mammalian meat oligosaccharide. This evinces that it is applied biophysics, rather than biochemistry that can solve the alpha gal allergy problem. The biochemical model of allergy evaluation and management has served the public well; its replacement is by no means implied by the introduction of SAAT. Implementation of this neuro-electric approach expands the therapeutic horizons of medicine. There is no disputing that electromagnetic forces can influence chemical processes. Prior to SAAT, a neurological paradigm for allergy has not been a viable consideration. Auricular acupuncture's neurological and embryologic basis lends to SAAT's apparent capability to reset a neuro-electric (bioenergetic) dysfunction of the liver, which manifests as allergy. Electrical detection and stimulation of ear acupuncture points-specific to each patient, appears to arrest the reaction between the offending allergen and its corresponding antibodies, in 3 weeks or less. The astonishing results and great convenience of SAAT, combined with its extremely low risk and cost, offers sufferers of alpha gal allergy an immediate opportunity for help from credentialed, experienced, and skilled practitioners, and supports the demand for large-scale clinical research studies.

\section{Disclosure Statement}

All the authors do not have any possible conflicts of interest.

\section{References}

[1] Van Nunen SA, O'Connor KS, et al. An association between tick bite reactions and red meat allergy in humans. Med J Aust. 2009 May 4; 190 (9): 510-1.

[2] Platts-Mills TA, Schuyler AJ, et al. Review: Delayed Anaphylaxis Involving IgE to Galactose-alpha-1,3-galactose. Curr Allergy Asthma Rep. 2015 Apr; 15 (4): 12.

[3] Commins SP, Satinover SM, et al. Delayed anaphylaxis, angioedema, or urticaria after consumption of red meat in patients with $\mathrm{IgE}$ antibodies specific for galactose-alpha-1,3-galactose. Allergy Clin Immunol. 2009 Feb; 123 (2): 426-33.

[4] Chabris, C, Simons, D. The invisible gorilla: And other ways our intuitions deceive us. Crown Publishers/Random House, 2010.

[5] Jutel M, Kosowska A, Smolinska S. Allergen Immunotherapy: Past, Present, and Future. Allergy Asthma Immunol Res. 2016; 8 (3): 191-197.

[6] Ring, J, Gutermuth, J. 100 years of hyposensitization: history of allergen-specific immunotherapy (ASIT). Allergy 2011 Jun; 66 (6): 713-24.

[7] Burks, A. Wesley et al. Treatment for food allergy. Journal of Allergy and Clinical Immunology, Volume 141, Issue 1, 1-9.

[8] American Academy of Allergy, Asthma \& Immunology. www.aaaai.org/conditions-and-treatments/library/allergy-libra ry/alpha-gal.

[9] Commins SP, Platts-Mills TA. Delayed anaphylaxis to red meat in patients with $\operatorname{IgE}$ specific for galactose alpha-1,3-galactose (alpha-gal). Curr Allergy Asthma Rep. 2013; 13 (1): 72-7.

[10] Wilson JM, Schuyler AJ, et al. Review Galactose- $\alpha-1,3-$ Galactose: Atypical Food Allergen or Model IgE Hypersensitivity? Curr Allergy Asthma Rep. 2017 Jan; 17 (1): 8.

[11] Yu W, Freeland DMH, Nadeau KC. Food allergy: immune mechanisms, diagnosis and immunotherapy. Nat Rev Immunol. 2016; 16 (12): 751-765. doi: 10.1038/nri.2016.111.

[12] Cohen, Sheldon G. Food allergens: Landmarks along a historic trail. Journal of Allergy and Clinical Immunology, Volume 121, Issue 6, 1521-1524.

[13] Sampson HA, Albergo R. Comparison of results of skin tests, RAST, and doubleblind, placebo-controlled food challenges in children with atopic dermatitis. J Allergy Clin Immunol 1984; 74: $26 \mathrm{e} 33$.

[14] Lopez CM, Mendez MD. Food Allergies. [Updated 2019 Oct 30]. In: Stat Pearls [Internet]. Treasure Island (FL): StatPearls Publishing; 2019 Jan-. Available from: https://www.ncbi.nlm.nih.gov/books/NBK482187/.

[15] Gellerstedt M., Bengtsson U., Niggemann B. Methodological issues in the diagnostic work-up of food allergy: a real challenge. J Investig Allergol Clin Immunol. 2007; 17: 350356. 
[16] Loh W, Tang MLK. The Epidemiology of Food Allergy in the Global Context. Int J Environ Res Public Health. 2018; 15 (9): 2043.

[17] Chinthrajah RS, Tupa D, Prince BT, Block WM, Rosa JS, Singh AM, Nadeau K. Diagnosis of Food Allergy. Pediatr Clin North Am. 2015 Dec; 62 (6): 1393-408.

[18] Igea JM. The history of the idea of allergy. Allergy 2013; 68: 966-973.

[19] Kanagaratham C, Sallis BF, Fiebiger E. Experimental Models for Studying Food Allergy. Cell Mol Gastroenterol Hepatol. 2018; 6 (3): 356-369.

[20] Burr, HS, Blueprint for immortality; the electric patterns of life. Neville Spearman, 1972

[21] World Health Organization Mondiale De La Sante. Report of the Working Group on Auricular Acupuncture Nomenclature. Lyon, France, 28-30 November 1990. WHO/TRM/91.2

[22] Soliman, N. A Comparison Study of the Effectiveness of SAAT (Soliman's Auricular Allergy Treatment) Approach and Nogier's Allergy Treatment Technique. Medical Acupuncture, Volume 26, Number 3, 2014.

[23] Zhou Q, Gai S, Lin N, et al. Power spectral differences of electrophysiological signals detected at acupuncture points and non-acupuncture points. Acupuncture \& Electro-Therapeutics Research. 2014; 39 (2): 169-181.

[24] Mercante B, Deriu F, Rangon C. Auricular Neuromodulation: The Emerging Concept beyond the Stimulation of Vagus and Trigeminal Nerves. Medicines. 2018; 5 (1): 10.

[25] Badran, B. W., Yu, A. B., Adair, D., Mappin, G., DeVries, W. H., Jenkins, D. D., George, M. S., Bikson, M. Laboratory Administration of Transcutaneous Auricular Vagus Nerve Stimulation (taVNS): Technique, Targeting, and Considerations. J. Vis. Exp. (143)

[26] Hosakoppal SS, Bryce PJ. Transplant-acquired food allergy: current perspectives. J Asthma Allergy. 2017; 10: 307-315.

[27] Penfield, Boldery. Penfield W, Boldery E. Somatic motor and sensory representation in the cerebral cortex of man as studied by electrical stimulation. Brain 1937; 60: 389-443.
[28] Zohar Tal Ran Geva Amir Amedi Positive and Negative Somatotopic BOLD Responses in Contralateral Versus Ipsilateral Penfield Homunculus Cerebral Cortex, Volume 27, Issue 2, 1 February 2017, Pages 962-980.

[29] Oleson TD, Kroening RJ, Bresler DE. An experimental evaluation of auricular diagnosis: the somatotopic mapping or musculoskeletal pain at ear acupuncture points. Pain. 1980 Apr; 8 (2): 217-29.

[30] Nihashi, T., Kakigi, R., Okada, T., Sadato, N., Kashikura, K., Kajita, Y., \& Yoshida, J. (2002). Functional magnetic resonance imaging evidence for a representation of the ear in human primary somatosensory cortex: Comparison with magnetoencephalography study. NeuroImage, 17 (3), $1217-1226$.

[31] Romoli, M., Allais, G., Airola, G. et al. Ear acupuncture and fMRI: a pilot study for assessing the specificity of auricular points. Neurol Sci (2014) 35 (Suppl 1): 189.

[32] Jones SR, Carley S, Harrison M. An introduction to power and sample size estimation. Emergency Medicine Journal 2003; 20: 453-458.

[33] Liebell, D. The Science of Auricular Microsystem Acupuncture: Amygdala Function in Psychiatric, Neuromusculoskeletal, and Functional Disorders. Medical acupuncture 313 (2019): 157-163.

[34] Excessive endoscopy) Rubenstein JH, Pohl H, Adams MA, et al. Overuse of Repeat Upper Endoscopy in the Veterans Health Administration: A Retrospective Analysis. The American Journal of Gastroenterology. 2017; 112 (11): 1678-1685.

[35] Seematter-Bagnoud L, Vader JP, Wietlisbach V, Froehlich F, Gonvers JJ, Burnand B. Overuse and underuse of diagnostic upper gastrointestinal endoscopy in various clinical settings. International Journal for Quality in Health Care: Journal of The International Society for Quality in Health Care. 1999; 11 (4): 301-308.

[36] Zamosky L. GERD: rising healthcare costs spark new debate, guidelines. Revised standards designed to save system money, prevent overuse of diagnostics. Medical Economics. 2013; 90 (10): 48 .

[37] H. A. Sampson. Invited review article, Food allergy: Past, present and future. Allergology International 65 (2016). 